\title{
Lifetime assessment of the process-dependent material properties of additive manufactured AISi10Mg under low-cycle fatigue loading
}

\author{
Carl Fischer ${ }^{1,2, *}$ and Christoph Schweizer ${ }^{1, * *}$ \\ ${ }^{1}$ Fraunhofer Institute for Mechanics of Materials IWM, Woehlerstrasse 11, 79108 Freiburg, Germany \\ ${ }^{2}$ Offenburg University of Applied Sciences, Badstrasse 24, 77652 Offenburg, Germany
}

\begin{abstract}
Systematic low-cycle fatigue (LCF) experiments are carried out on additive manufactured AlSi10Mg specimens for several material conditions with varying layer thickness, heat treatment, building direction and surface quality. The deformation behaviour depends significantly on the heat treatment. It is outlined that the process control and heat treatment can produce fatigue properties comparable with the cast material, whereby an as-built specimen surface leads to a lifetime reduction in all cases. The experiments are accompanied with detailed metallo- and fractographic investigations. For all tested LCF specimens, the defect type and the failure origin defect size are characterized in terms of the $\sqrt{\text { area }}$ parameter by using scanning electron microscopy. The failure of the specimen is mostly caused by lack of fusion surface or near-surface defects, whereby the defect size is determined by the SLM process parameters, such as building direction, surface quality and layer thickness. On the basis of the experimental data and the observed defects, a mechanism-based, deterministic lifetime model is developed and adapted to the specific damage mechanisms of the additive manufactured AlSi10Mg alloy.
\end{abstract}

\section{Introduction}

The selective laser melting (SLM) process stands at the beginning of a major development for many medical and aerospace applications and also in many other sectors of industry [1]. Although some additive manufacturing systems are technologically similar, parameter sets for laser power, laser focus, scanning strategy and layer thickness are very different and can be freely chosen by the operator [2]. Especially for precipitation hardening materials such as the aluminium alloy AlSi10Mg, the mechanical material and component properties vary locally [1-3]. To design and merchandise highly stressed additive manufactured components, manufacturers must understand the relationships between the process and the resulting microstructure-dependent material properties [1].

The primary objective in the qualification of additive manufactured materials is to achieve the optimum in component density $[4,5]$. For this purpose, a wide matrix of parameter sets are systematically studied to obtain the lowest-possible porosity $[4,6]$. In order to characterize the mechanical properties, usually micro-hardness and tensile tests are carried out [6]. An overview over the tensile properties of additive manufactured aluminium alloys is given, e.g. in $[1,3,6]$. For dynamically loaded applications, high-cycle fatigue ( $\mathrm{HCF}$ ) tests are performed to determine the fatigue strength [7-10]. Current research is dealing with the material characterization for a wide spectrum of loading conditions, so that in addition to HCF, the

\footnotetext{
*e-mail: carl.fischer@iwm.fraunhofer.de

**e-mail: christoph.schweizer@iwm.fraunhofer.de
}

low-cycle fatigue (LCF) properties of additive materials are investigated more intensively [11-13].

For additive manufactured aluminium materials it is reported in literature, that the fatigue properties are very sensitive to defects or the defect structure $[3,6,7]$, the rough surface quality $[6,11]$ and residual stresses [6]. The manufacturing process yields preferentially to gas pores and lack of fusion inclusions, which have a major influence on the mechanical properties and therefore have to be avoided or at least reduced [1, 2]. In [3], the fatigue strength of additive manufactured and cast aluminium alloys is correlated with an initial defect size in terms of the $\sqrt{\text { area }}$ parameter from Murakami [14]. The parameter $\sqrt{\text { area }}$ itself denotes the size of an internal arbitrarily shaped crack [14]. The large material scatter in HCF results is assessed with $\sqrt{\text { area }}$ and extreme value statistics, where the defect population and defect size distribution of whole specimens are applied by computer tomography scan measurements in order to predict lifetimes and also the failure probability of additive manufactured AlSi10Mg $[15,16]$. The $\sqrt{\text { area }}$ concept is also adapted in $[9,11]$ for the LCF lifetime prediction of additive manufactured AlSi10Mg by using elastic-plastic fracture mechanics based on the cyclic $J$-integral $\Delta J$. The identical fracture mechanics approach is applied for the fatigue life prediction of additive manufactured Ti-6Al-4V in [12] and 316L stainless steel in [12, 13].

In this work, systematic LCF tests are carried out for a selective laser melted (SLM) AlSi10Mg alloy. The aim of the investigations is to be able to understand and evaluate the connections between the process-dependent mi- 
crostructure, the defect structure and the LCF lifetime properties. The defect type and the failure origin defect size are examined by means of $\sqrt{\text { area }}$ from high resolution scanning electron microscopy fracture surface images. With the obtained material parameters from the LCF tests and the initial defect sizes, the lifetimes are assessed using a mechanism-based deterministic lifetime model.

\section{Investigated material conditions and experimental test set-up}

An EOS M400 system at the Fraunhofer EMI institute was used for additive manufacturing of the AlSi10Mg aluminium samples. The system is equipped with a $1 \mathrm{~kW}$ YLR series CW laser with a wavelength of $1070 \mathrm{~nm}$. The chemical composition of the EOS AlSi10Mg powder is given in [17]. The samples are manufactured under argon atmosphere with a maximum oxygen content of $1.2 \%$ on a substrate plate, which was preheated to $165^{\circ} \mathrm{C}$. Five sets of process parameters given in table 1 are used to produce the specimens. The specimens with a layer thickness of $90 \mu \mathrm{m}$ are produced in a $67^{\circ}$ rotating stripe strategy using the parameter sets provided from the system manufacturer. For the reduced layer thickness of $30 \mu \mathrm{m}$ the parameter set was developed by the Fraunhofer EMI and cannot be given in detail due to confidentiality reasons.

Table 1: Characterized material conditions.

\begin{tabular}{ccc}
\hline Process & Layer thickness & Heat treatment \\
\hline 1 & $90 \mu \mathrm{m}$ & none \\
\hline 2 & $90 \mu \mathrm{m}$ & $2 \mathrm{~h}$ at $300{ }^{\circ} \mathrm{C}$ \\
\hline 3 & $90 \mu \mathrm{m}$ & $\begin{array}{c}\text { Annealing: } 1 \mathrm{~h} \text { at } 525^{\circ} \mathrm{C} \\
\text { Quenching: } 60{ }^{\circ} \mathrm{C} \text { water } \\
\text { Ageing: } 6 \mathrm{~h} \text { at } 165^{\circ} \mathrm{C}\end{array}$ \\
\hline 4 & $30 \mu \mathrm{m}$ & none \\
\hline 5 & $30 \mu \mathrm{m}$ & $2 \mathrm{~h}$ at $300^{\circ} \mathrm{C}$ \\
\hline
\end{tabular}

From the first manufacturing process, the material with a powder layer thickness of $90 \mu \mathrm{m}$ is characterised without additional heat treatment (referred to as material condition $90 \mu \mathrm{m}$ ). The material from the second manufacturing process (named as $90 \mu \mathrm{m}+\mathrm{T} 5$ ) is subjected to a stress relief heat treatment for two hours at $300{ }^{\circ} \mathrm{C}$, which is intended to reduce anisotropies caused by the manufacturing process [17]. The stress relief heat treatment reduces the hardness from $121 \mathrm{HV} 10$ to $84 \mathrm{HV} 10$. The influence of the reduced layer thickness of $30 \mu \mathrm{m}$ is examined for identical heat treatment conditions for the material conditions $30 \mu \mathrm{m}$ and $30 \mu \mathrm{m}+\mathrm{T} 5$. Since the casting counterpart is usually exposed to a strength-enhancing T6 heat treatment [18], the influence of a T6 heat treatment on the additive manufactured $\mathrm{AlSi} 10 \mathrm{Mg}$ alloy is investigated in this work (named as $90 \mu \mathrm{m}+\mathrm{T} 6$ ).

After the SLM process, mechanical properties similar to the T6 condition of cast components are reported in [17]. For this purpose, a T6 heat treatment is not recommended for laser sintering components and instead a stress relief heat treatment for two hours at $300^{\circ} \mathrm{C}$ is suggested by the system manufacturer to reduce or eliminate feasible anisotropic properties due to the layer structure by suitable thermal post heat treatments [17]. However, in order to investigate the extent to which positive effects on the mechanical properties can be achieved by adjusting the material's microstructure, a batch of additive manufactured samples was subjected to an additional strength-enhancing T6 heat treatment. In literature, strongly varying periods for the solution heat treatment are specified for SLM AlSi10Mg alloys (cf. [7, 8, 19-22]). Therefore, the influence of the solution heat treatment time on the microstructure was investigated first. Figure 1 shows the evolution of the microstructure after solution heat treatment compared to the initial state. In the initial state, the eutectic silicon (Si) regions are existent in fine, coherent cell structures. By applying a solution heat treatment, the silicon phase first becomes spheroidal in shape and then coarsens with increasing time, which is also observed in [22]. Furthermore, the needle-like ferrous $\beta-\mathrm{Al}_{5} \mathrm{FeSi}$ phase is formed [20-24]. According to [21, 22], the solution heat treatment also forms beneficial $\mathrm{Mg}_{2} \mathrm{Si}$ precipitations, which are largely responsible for the increase in strength during the T6 heat treatment. On the basis of the preliminary investigations, a short solution heat treatment time of one hour was chosen, as a fine microstructure with spherical phases was achieved. In addition, the formation of the detrimental $\beta-\mathrm{Al}_{5} \mathrm{FeSi}$ is impeded, which could lead to high stress concentrations due its platelet-like shape [25] and is thus prone to fracture [26] as well as crack nucleation and propagation reducing the fatigue lifetime [25, 27].

Figure 2 shows the microstructure of the additive manufactured AlSi10Mg alloy from the first manufacturing process in the initial $90 \mu \mathrm{m}$ material condition. The metallographic section is prepared with Murakami etching for $15 \mathrm{~s}$ in order to visualise the individual layers and the heataffected zones. The microstructure contains isolated gas pores as well as some lack of fusion areas due to incompletely melted powder during the manufacturing process.

In order to investigate the influence of the building direction, the specimens are manufactured in three orientations of $0^{\circ}$ (specimen axis parallel to manufacturing layer), $45^{\circ}$ (specimen axis diagonal to manufacturing layer) and $90^{\circ}$ (specimen axis perpendicular to manufacturing layer). Due to rapid cooling rates during the manufacturing process, residual stresses are introduced in the material, so that especially large-volume, horizontally oriented specimens are prone to premature crack formation $[2,28]$. In order to minimize this problem, additional support structures acting as thermal bridges for improved heat dissipation and also short specimen geometries are applied [28]. $80 \mathrm{~mm}$ long, smooth cylindrical specimens with $9 \mathrm{~mm}$ gauge length and a diameter of $8 \mathrm{~mm}$ were sampled and polished from the raw material (see figure $3 \mathrm{a}$ ). Some of the $45^{\circ}$ oriented specimens were also manufactured with as-built surface (see figure $3 b$ ).

Strain-controlled LCF tests were performed to experimentally characterize the process-dependent mechanical properties of the additive manufactured aluminium alloy. The LCF specimens were tested in ambient air with a 


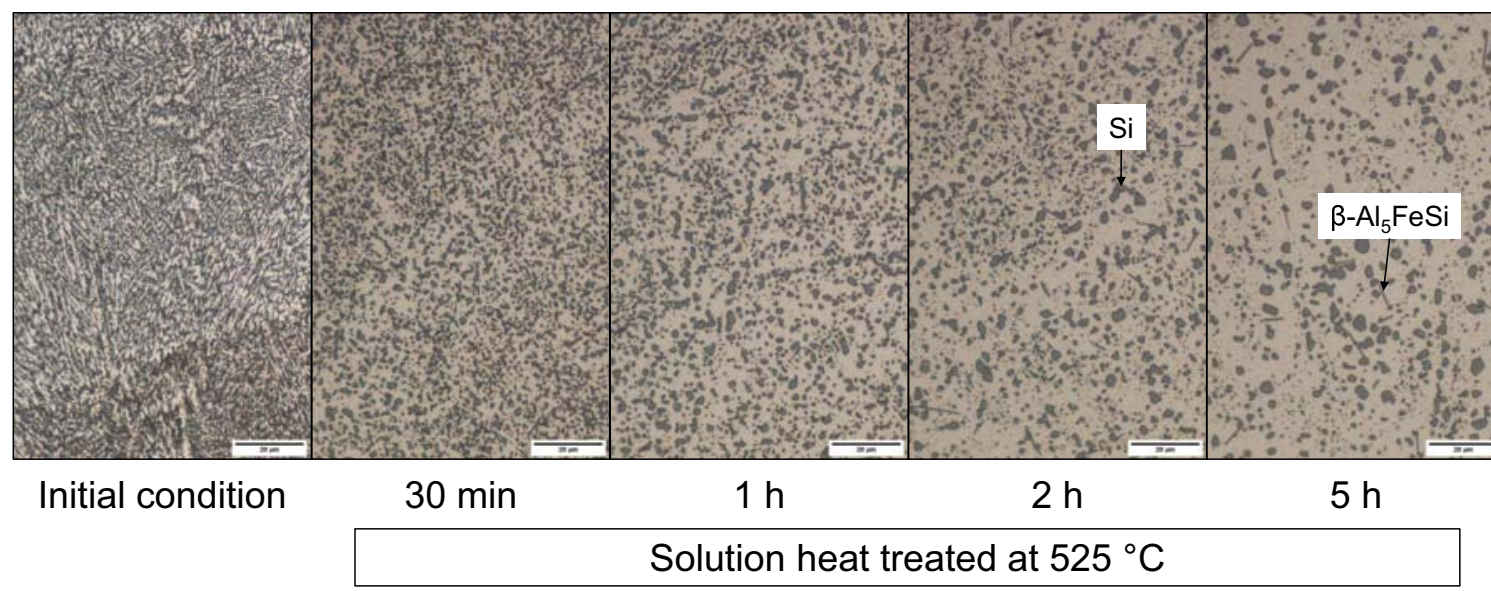

Figure 1: Evolution of the microstructure with solution heat treatment time at $525^{\circ} \mathrm{C}$ compared to the initial condition (Murakami etched light microscopy images).

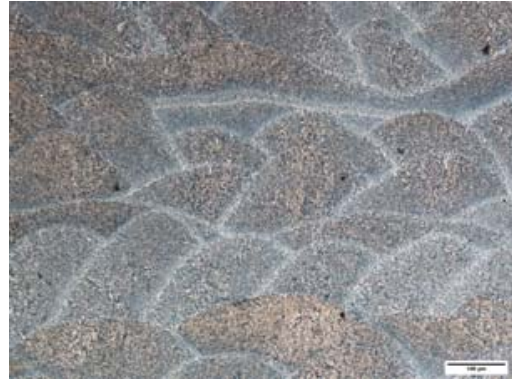

Figure 2: Light microscopy image of the $90 \mu \mathrm{m}$ SLM AlSi10Mg microstructure after $15 \mathrm{~s}$ Murakami etching.

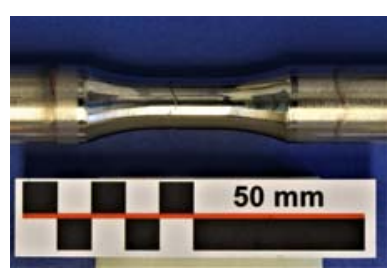

(a)

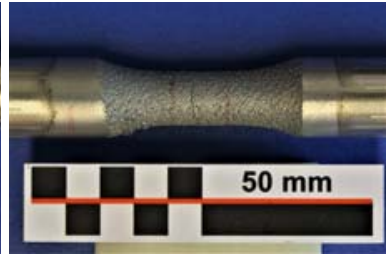

(b)
Figure 3: (a) Polished and (b) as-built surface specimen conditions (tested specimens).

$100 \mathrm{kN}$ electromechanical Instron 8862 universal testing machine. A capacitive extensometer with a calibrated gauge length of $8 \mathrm{~mm}$ from Epsilon Technology Corp is employed for measuring and controlling the axial strain. The temperature is measured with a type $\mathrm{K}$ ribbon thermocouple placed in the middle of the gauge length. A LabView software application is used to enable the test conditions and for data collection. All LCF tests are carried out in a triangular cycle with a constant strain rate of $\mathrm{d} \varepsilon / \mathrm{d} t=10^{-3} 1 / \mathrm{s}$ and fully reversed loading $\left(R_{\varepsilon}=-1\right)$ and the mechanical strain amplitudes are chosen to $\varepsilon_{\mathrm{a}}^{\text {mech }}=$ $0.3 \%, 0.4 \%$ and $0.5 \%$.

\section{Results of the low-cycle fatigue tests}

\subsection{Deformation behaviour at half lifetime}

The influence of the building direction, layer thickness and heat treatment on the deformation behaviour is explained in Figure 4 by means of stabilized stress-strain hysteresis loops at half lifetime $N_{\mathrm{A}} / 2$ for a mechanical strain amplitude of $\varepsilon_{\mathrm{a}}^{\text {mech }}=0.5 \%$. The hysteresis loops of the $90 \mu \mathrm{m}$ material condition in figure $4 \mathrm{a}$ show a slight tensioncompression asymmetry, which is most pronounced for the building direction of $90^{\circ}$ and the $45^{\circ}$ oriented specimen in as-built condition. The stress response as well as the elastic material stiffness of the $45^{\circ}$ as-built specimen are significantly lower than for the machined and polished specimens. The stress relief heat treatment diminishes the observed stress asymmetry and leads to a deformation behaviour which is independent of the building direction and the surface condition for the $90 \mu \mathrm{m}+\mathrm{T} 5$ material (see figure 4b). However, the heat treatment significantly reduces the overall mechanical strength, whereby the cyclic hardening behaviour is also decreased.

Although the T6 heat treatment has a strong influence on the microstructure morphology of the investigated SLM AlSi10Mg alloy (see figure 1), the heat treatment shows a negligible effect on the hysteresis loops in comparison to the $90 \mu \mathrm{m}$ condition as depicted in figure 4c. Consequently, the $90 \mu \mathrm{m}+$ T6 material condition is comparable to the initial $90 \mu \mathrm{m}$ material condition, which received no further heat treatment after manufacturing. It has to be mentioned, that this conclusion only holds for small strains, since tensile tests on the $90 \mu \mathrm{m}+\mathrm{T} 6$ condition revealed a decrease in hardening for T6 compared to the initial $90 \mu \mathrm{m}$ condition. The cyclic hardening behaviour as well as the ductility are gently reduced by the peak enhancing T6 heat treatment. Unfortunately, no specimens of the T6 material condition were available with as-built surface.

Figure $4 \mathrm{~d}$ illustrates for the $30 \mu \mathrm{m}$ material condition, that a reduced powder layer thickness does not affect the hysteresis loops considerably (compare with figure $4 a$ ). 


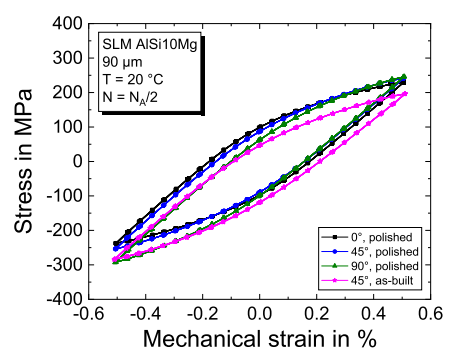

(a)

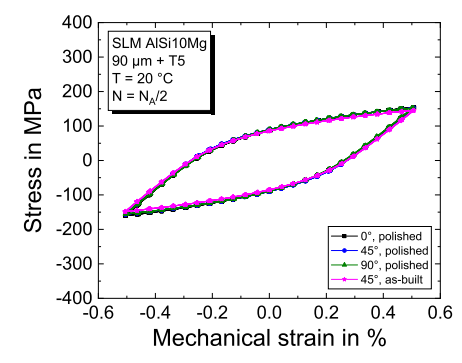

(b)

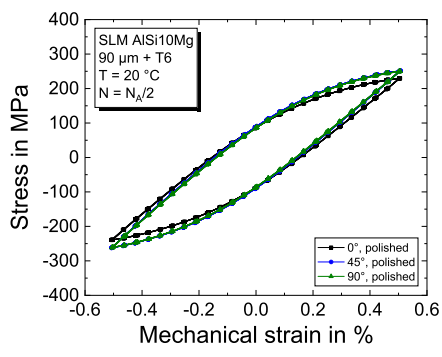

(c)

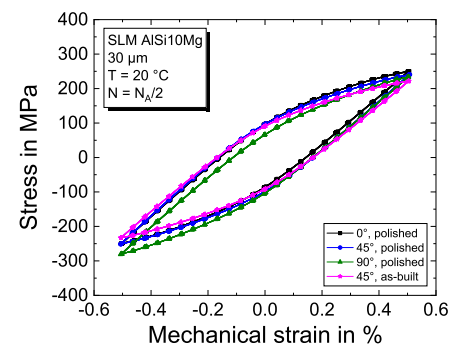

(d)

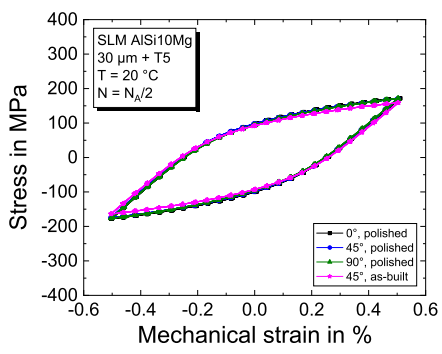

(e)

Figure 4: Deformation behaviour of half life hysteresis loops for a mechanical strain amplitude of $\varepsilon_{\mathrm{a}}^{\text {mech }}=0.5 \%$ for the investigated material conditions: (a) $90 \mu \mathrm{m}$, (b) $90 \mu \mathrm{m}+\mathrm{T} 5$, (c) $90 \mu \mathrm{m}+\mathrm{T} 6$, (d) $30 \mu \mathrm{m}$ and (e) $30 \mu \mathrm{m}+\mathrm{T} 5$.

The tension-compression asymmetry is also apparent and most pronounced for the $90^{\circ}$ oriented specimen. Moreover, the resulting stresses are somewhat reduced for the as-built specimen surface. The reduced layer thickness of $30 \mu \mathrm{m}$ in combination with the stress relief heat treatment (see figure $4 \mathrm{e}$ ) has hardly any effect on the mechanical strength in comparison to the $90 \mu \mathrm{m}+\mathrm{T} 5$ material condition.

\subsection{Lifetime behaviour}

In addition to the results shown in figure 4, further LCF tests with mechanical strain amplitudes of $\varepsilon_{\mathrm{a}}^{\text {mech }}=$ $0.3 \%$ and $0.4 \%$ are performed. The lifetimes of all LCF tests for the different material conditions are presented in figure 5. If possible, the lifetimes are determined by a $5 \%$ drop of the maximum stress as recommended in [29]. For classification and comparability reasons, the obtained test results are plotted together with LCF lifetimes from [11], which correspond to a not further heat-treated SLM AlSi10Mg alloy with two different layer thicknesses and two building directions (horizontal and vertical), which were manufactured in three processes (process 1 to process 3 ). For all considered material conditions, independent of heat treatment and the used powder layer thickness, there is no markable influence of the building direction on the lifetime. The $90 \mu \mathrm{m}$ condition in figure $5 \mathrm{a}$ exhibits the shortest lifetimes. Compared to the literature data from [11], the lifetimes are found at the lower scatter band. The as-built surface condition reduces the lifetime significantly, whereby this trend seems to be more pronounced at lower mechanical strains. Although the stress relief heat treatment reduces the strength of the material (cf. figure $4 b$ ) noticeably, the lifetime behaviour in figure $5 \mathrm{~b}$ is enhanced in comparison to the initial material condition. The detrimental influence on the lifetime due to as-built surface conditions is also significantly attenuated by the subsequent stress relief heat treatment. The T6 heat treatment does not have a significant effect on the lifetime (cf. figures 5a and 5c). Please note, that no as-built specimens were available for this material condition. The reduced powder layer thickness of $30 \mu \mathrm{m}$ has a positive effect on the lifetime (see figures $5 \mathrm{~d}$ and $5 \mathrm{e}$ ), especially at high mechanical strains. The as-built surface condition has a detrimental influence on the lifetime, which is also reduced by the heat treatment. The obtained results correspond very well with the experimental tests of process 3 from [11], where the layer thickness is also $30 \mu \mathrm{m}$. The longest lifetimes are achieved with a powder layer thickness of $30 \mu \mathrm{m}$ and subsequent stress relief heat treatment (see figure $5 \mathrm{e}$ ).

In figure 5f, the SLM lifetimes of the polished specimens are compared to similar cast aluminium alloys from the literature [18, 30-35]. The scatter of the cast aluminium materials is very pronounced. The lifetimes of the SLM AlSi10Mg alloy are found at the upper scatter band of the cast aluminium alloys for the mechanical strain amplitudes of $\varepsilon_{\mathrm{a}}^{\text {mech }}=0.4 \%$ and $0.5 \%$. At a mechanical strain amplitude of $\varepsilon_{\mathrm{a}}^{\text {mech }}=0.3 \%$, the lifetimes tend to be shorter, since the level of the mechanical load has a more pronounced influence on the lifetime behaviour of the SLM alloy than for the cast aluminium materials. A T5 heat treatment results in the shortest lifetimes for 


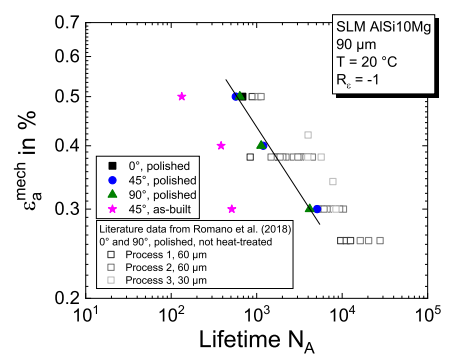

(a)

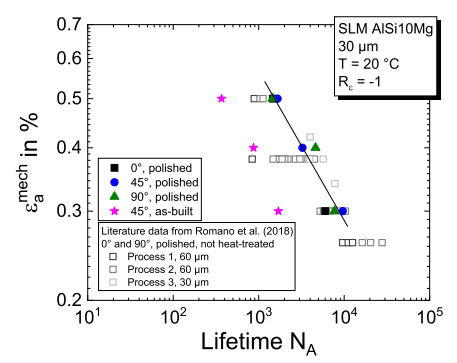

(d)

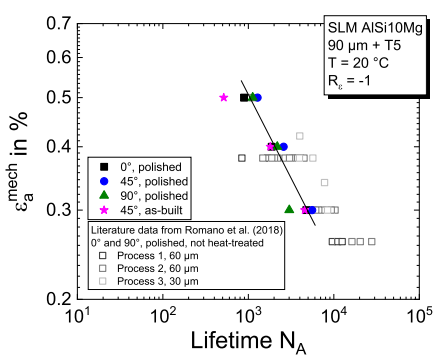

(b)

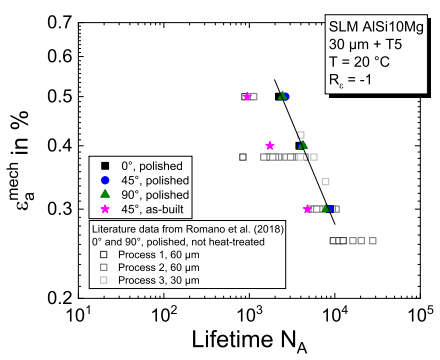

(e)

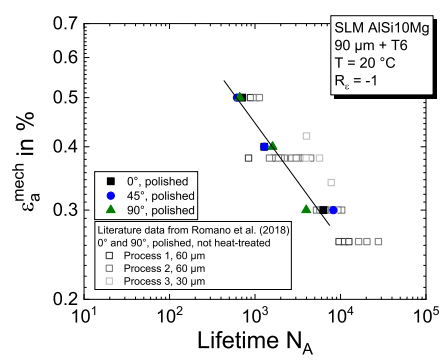

(c)

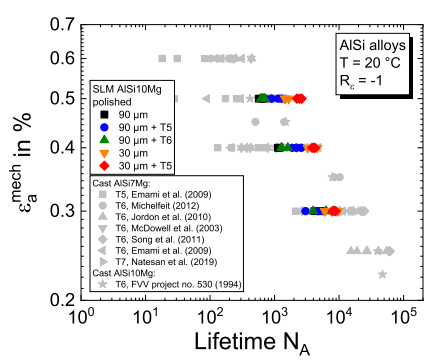

(f)

Figure 5: Lifetime behaviour of the investigated material conditions in comparison to LCF tests from [11]: (a) $90 \mu \mathrm{m}$, (b) $90 \mu \mathrm{m}$ and T5, (c) $90 \mu \mathrm{m}$ and T6, (d) $30 \mu \mathrm{m}$ and (e) $30 \mu \mathrm{m}$ and T5. (f) Comparison of SLM lifetimes of polished specimens with literature data from [18, 30-35] for similar cast aluminium alloys.

the AlSi7Mg cast material. The lifetime behaviour is enhanced with a T6 heat treatment, whereby further artificially over ageing to $\mathrm{T} 7 \mathrm{~h}$ hs no significant impact. In contrast to the observations for the cast materials, a T5 heat treatment of the SLM AlSi10Mg alloy leads to prolonged lifetimes. The T6 heat treatment does not improve the lifetime behaviour in case of the additive manufactured AlSi10Mg alloy. To sum up, the lifetimes of the SLM AlSi10Mg alloy are comparable to those of the cast aluminium alloys.

\section{Defect structure analysis}

By means of high-resolution scanning electron microscopy images of the fracture surfaces, the defect structure and the failure origin defect size of all LCF specimens is characterised in terms of the $\sqrt{\text { area }}$ parameter from Murakami [14]. Figure 6 shows exemplary defect sizes and defect types at the failure origin. The failure of the specimens is caused by single lack of fusion surface or near-surface defects. Although all specimens contained gas porosities, gas pores were not found to act as detrimental crack initiation sites. Only three specimens failed due to internal defects, which were also induced by lack of fusion. In some cases, multiple defects or defect clusters are observed. Especially the as-built surface specimens are very sensitive to multiple crack initiation. A considerable number of defects are found at surface and the existing roughness leads to complex surface profiles and exposed defect locations, which are very favourable for crack initiation.
The defect size is determined using the image processing software Fiji [36]. Only the defect size that led to the failure of the specimen is evaluated. However, one or more secondary cracks are present on almost all fracture surfaces. The defect size distribution by means of $\sqrt{\text { area }}$ is presented in figure 7 for different building directions and surface conditions. The building direction of the samples has a noticeable influence on the defect size, as the horizontal $\left(0^{\circ}\right)$ and vertical $\left(90^{\circ}\right)$ oriented specimens tend to have larger initial defects. The defect size is generally reduced by the lower layer thickness of $30 \mu \mathrm{m}$. For the horizontally oriented specimens a large scatter in defect size is observed. The smallest defects are found for the $45^{\circ}$ specimens independent of the powder layer thickness. The overall defect size is increased again for the $90^{\circ} \mathrm{spec}-$ imens, whereas two outliers are found for the $90 \mu \mathrm{m}+\mathrm{T} 5$ condition. Also one outlier is observed for the $30 \mu \mathrm{m}$ material condition, which is caused by a large internal defect. The defect size is significantly larger for the as-built specimens, whereby a smaller layer thickness also has a positive effect on the defect size.

\section{Mechanism-based deterministic lifetime modelling}

In this section, the obtained lifetimes from section 3.2 are assessed by using a mechanism-based lifetime model. In [37], two cast aluminium alloys used for cylinder heads and pistons in combustion engines were extensively characterised and the fracture mechanics based 


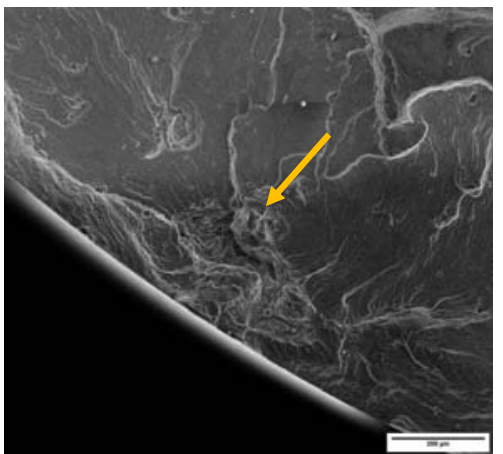

(a)

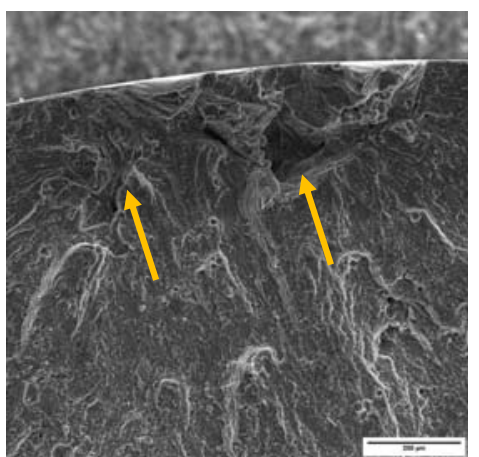

(b)

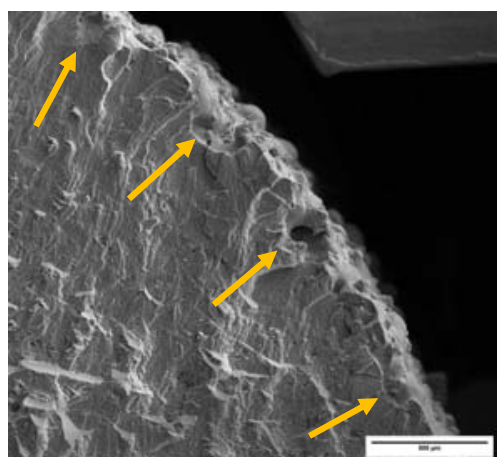

(c)

Figure 6: Examples for defect sizes and defect types at failure origin: (a) single lack of fusion surface defect ( $90 \mu \mathrm{m}$ specimen), (b) lack of fusion cluster $(90 \mu \mathrm{m}$ specimen) and (c) typical multiple crack initiation at as-built surfaces ( $30 \mu \mathrm{m}$ specimen).

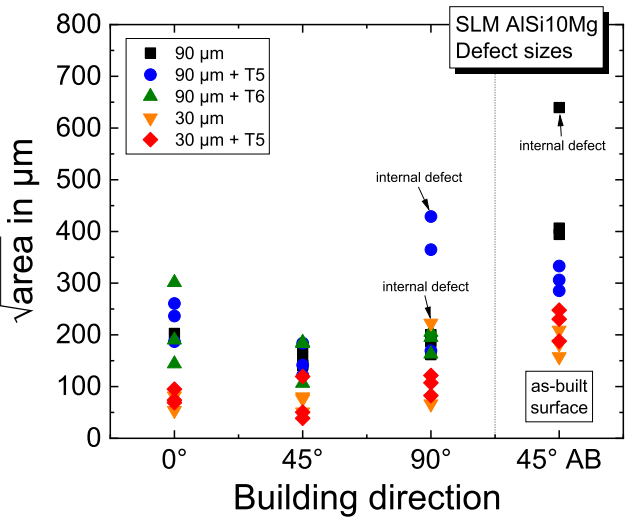

Figure 7: Defect sizes in dependence of building direction, layer thickness and surface condition.

damage parameter $D_{\mathrm{TMF}}$,rittle was developed, which accounts the temperature-dependent influence of the mechanical strength (i.e. cyclic yield stress $\sigma_{\mathrm{CY}}$ ) on the damage mechanism more accurately:

$$
D_{\mathrm{TMF}, \text { brittle }}=d_{\mathrm{n}^{\prime}} Z_{\mathrm{D}} \sigma_{\mathrm{CY}}^{q} F(t, \sigma, T) .
$$

The pre-factor $d_{\mathrm{n}^{\prime}}$ depends on the hardening behaviour of the material by means of the cyclic Ramberg-Osgood [38] hardening exponent $n^{\prime}$. Both for plane strain and plane stress states, $d_{\mathrm{n}^{\prime}}$ values are tabulated in [39], whereby a third order polynomial function for plane strain is published in [40]. Furthermore in equation (1), $Z_{\mathrm{D}}$ denotes the damage parameter from [41], $\sigma_{\mathrm{CY}}$ the cyclic yield stress at a plastic strain offset of $0.2 \%$ and setting the exponent to $q=0.2$ has turned out to be a good choice for cast aluminium materials. The function $F(t, \sigma, T)$ (see e.g. in [42]) accounts for time-dependent effects and arbitrary stress-temperature histories. Since this work only considers room temperature LCF tests, $F=1$ holds for all tests. The damage parameter $D_{\mathrm{TMF}, \text { brittle }}$ is based on the $D_{\mathrm{TMF}}$ parameter from [43], which takes the form:

$$
D_{\mathrm{TMF}}=\frac{Z_{\mathrm{D}}}{\sigma_{\mathrm{CY}}} F(t, \sigma, T)
$$

$D_{\mathrm{TMF}}$ is modified accounting for the brittle fracture of hard inclusions (e.g. primary silicon particles or hard intermetallic phases) in a ductile matrix, which promotes sudden crack growth e.g. in aluminium-silicon alloys. Since brittle fracture is usually a stress-controlled process, the cyclic yield stress $\sigma_{\mathrm{CY}}$ appears in the numerator of $D_{\mathrm{TMF}, \text { brittle. }}$

A power-law correlation between the crack growth increment per cycle $\mathrm{d} a / \mathrm{d} N$ and the damage parameter $D_{\mathrm{TMF}, \text { brittle }}$ is assumed:

$$
\frac{\mathrm{d} a}{\mathrm{~d} N}=\beta\left(D_{\mathrm{TMF}, \text { brittle }} a\right)^{B}
$$

In $D_{\mathrm{TMF}, \text { brittle }} a$ from equation (3), $Z_{\mathrm{D}} a$ provides an estimate of the effective cyclic $J$-integral $\Delta J_{\text {eff }}$ for semicircular surface cracks:

$$
\Delta J_{\mathrm{eff}}=Z_{\mathrm{D}} a=\left(1.45 \frac{\Delta \sigma_{\mathrm{eff}}^{2}}{E}+2.4 \frac{\Delta \sigma \Delta \varepsilon^{\mathrm{pl}}}{\sqrt{1+3 n^{\prime}}}\right) a .
$$

The stress range $\Delta \sigma$, plastic strain range $\Delta \varepsilon^{\mathrm{pl}}$ and the cyclic hardening Ramberg-Osgood exponent $n^{\prime}$ are usually determined from stabilized hysteresis loops at half lifetime. For the effective stress range follows $\Delta \sigma_{\text {eff }}=$ $\sigma_{\max }-\sigma_{\text {op }}$ with the maximum stress $\sigma_{\max }$ and the crack opening stress $\sigma_{\text {op. }}$. Crack closure effects are taken into account by means of the analytical system of equations published in [44]. In order to assess internal defects, the solution for a penny-shaped crack in an infinite medium under mode I loading with the stress intensity factor given e.g. in [45] and the plastic solution from [46] is applied to cyclic loading:

$$
\Delta J_{\mathrm{eff}}=\left(\frac{4}{\pi} \frac{\Delta \sigma_{\mathrm{eff}}^{2}\left(1-v^{2}\right)}{E}+\frac{6}{\pi} \frac{\Delta \sigma \Delta \varepsilon^{\mathrm{pl}}}{\sqrt{1+3 n^{\prime}}}\right) a .
$$

According to [47], equation (4) is obtained from equation (5) by the multiplication of the pre-factors $4 / \pi$ and 
$6 / \pi$ with a squared correction factor of $1.12^{2}$ and inserting $v=0.3$ for the Poisson's ratio.

In LCF tests, the lifetime $N_{\mathrm{A}}$ is determined by crack initiation, subsequent short crack growth and the formation of a technical, ca. $1 \mathrm{~mm}$ long macroscopic crack. Especially in the LCF regime with high strain amplitudes, the crack initiation phase has no significant influence on the lifetime [37, 48-50]. The lifetime $N_{\mathrm{A}}$ is integrated from equation (3) by separation of variables from an initial crack length $a_{0}$ to a final crack length $a_{\mathrm{f}}$. Assuming that $D_{\mathrm{TMF}, \text { brittle }}$ does not change significantly over the number of cycles one obtains:

$$
N_{\mathrm{A}}=\frac{A}{D_{\mathrm{TMF}, \text { brittle }}^{B}},
$$

where

$$
A=\ln \left(\frac{a_{\mathrm{f}}}{a_{0}}\right) \quad \text { for } B=1,
$$

and:

$$
A=\frac{a_{\mathrm{f}}^{1-B}-a_{0}^{1-B}}{(1-B) \beta} \quad \text { for } B>1 .
$$

In order to assess the lifetimes in figure 5, the material properties $E, \sigma_{\mathrm{CY}}$ and $n^{\prime}$ are determined first from the stabilised hysteresis loops at half lifetime from figure 4. The pre-factor $d_{n^{\prime}}$ is calculated by applying the third order polynomial function given in [40]. Surface or near-surface defects are considered as semicircular surface cracks, therefore equation 4 is used for the calculation of $Z_{\mathrm{D}}$. For specimens where an internal defect resulted in failure, a penny-shaped crack geometry is assumed and equation 5 is applied.

The mechanism-based lifetime assessment in terms of the $D_{\mathrm{TMF}, \text { brittle }}$ parameter is presented in figure 8 . The im-

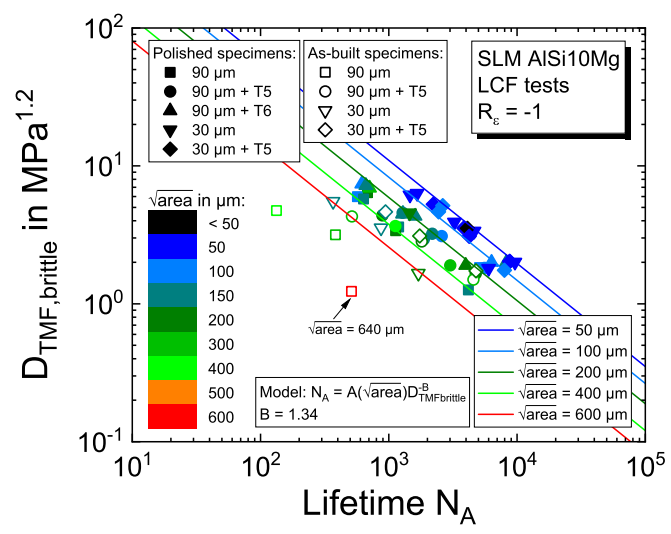

Figure 8: Lifetime assessment by means of $D_{\mathrm{TMF}, \text { brittle }}$ and influence of $\sqrt{\text { area }}$ on the lifetime curves.

pact of the initial defect size on the lifetime is indicated by the colour scale, where larger initial defects mostly lead to reduced lifetimes. This trend is especially evident in the case of as-built and not heat-treated $90 \mu \mathrm{m}$ specimens, where initial defect sizes larger than $600 \mu \mathrm{m}$ occur. In addition, the influence of varying sizes for $\sqrt{\text { area }}$ on the predicted lifetime curves is shown in figure 8 . To this end, $\sqrt{\text { area }}$ is converted either to a semicircular surface $a_{0}=\sqrt{\text { area }} \sqrt{2 / \pi}$ or to a penny-shaped initial crack length $a_{0}=\sqrt{\text { area }} / \sqrt{\pi}$. The exponent $B=1.34$, i.e. the slope of the lifetime model, is fitted to the experiments with the smallest initial defect size (i.e. polished $30 \mu \mathrm{m}$ specimens), because a minimum lifetime scatter is assumed and logically the slope of the lifetime curve should not be affected much by outliers. Afterwards, the factor $\beta$ is fitted with the defect sizes of the polished $30 \mu \mathrm{m}$ specimens from figure 7 according to equation (8) with the previous determined factor $B$ and a final crack length of $a_{\mathrm{f}}=a_{0}+1 \mathrm{~mm}$. With the obtained result for $\beta=2.4 \times 10^{-4} \mathrm{~m}^{1-B} /\left(\right.$ cycle $\left.\cdot \mathrm{MPa}^{1.2 B}\right)$, equation (8) is applied with the exemplary $\sqrt{\text { area }}$ sizes shown in figure 8 . Most of the LCF tests with defect sizes of $\sqrt{\text { area }}$ between $50 \mu \mathrm{m}$ and $400 \mu \mathrm{m}$ are well described with the corresponding curves. The lifetimes show a strong dependency on $\sqrt{\text { area. }}$ However, lifetimes of the as-built specimens are overestimated despite of the considered initial defect size.

The deterministic lifetime prediction is performed in figure 9 considering the initial defect size according to equation (8) in the pre-factor $A(\sqrt{\text { area }})$ with the previous determined parameters $B, \beta$ and $a_{\mathrm{f}}$. A probability density function is adjusted to the difference between the logarithmic experimental and predicted lifetimes. The given scatter band factor is calculated with the standard deviation $\sigma$ to $10^{2 \sigma}$ and corresponds to a $95 \%$ confidence interval. By

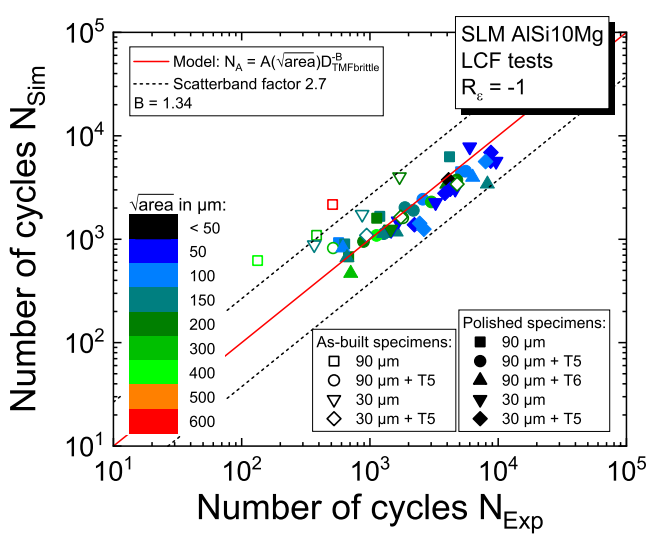

Figure 9: Deterministic lifetime prediction by means of $D_{\mathrm{TMF}, \text { brittle }}$ and the initial defect size $\sqrt{\text { area }}$.

considering the individual initial defect size $\sqrt{\text { area }}$ of each specimen in the mechanism-based, deterministic lifetime model, the determined scatter band factor is reduced from 4.4 to 2.7. However, the predicted lifetimes of the nonheat treated and as-built specimens are still overestimated. Thus, the effective defect size $\sqrt{\operatorname{area}_{\text {eff }}}[14]$ is taken into account, which allows an estimate for the effect defect size of irregular shaped defects by considering a smooth enveloping contour, for the interaction between a near- 
surface defect and the specimen's surface or for the interaction effect between two adjacent defects. The lifetime prediction in terms of the effective defect size $\sqrt{\text { area }_{\text {eff }}}$ is presented in figure 10. Please note, that the identical

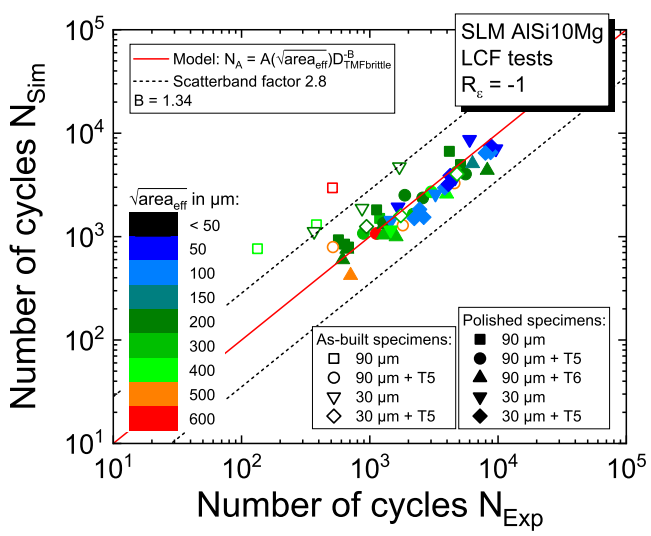

Figure 10: Deterministic lifetime prediction by means of $D_{\mathrm{TMF}, \text { brittle }}$ and the initial effective defect size $\sqrt{\operatorname{area}_{\mathrm{eff}}}$.

colour scale from figure 9 is used and due to larger effective defect sizes the symbol colour might change. The expectation to obtain an enhanced description of the experimental results is not fulfilled. The achieved scatter band by a factor of 2.8 is even slightly larger than in figure 9 . Especially the as-built specimens are found more closely at the upper scatter band or even further outside. The scatter band factor is slightly increased also for the other experiments.

\section{Discussion}

The cyclic hysteresis loops in figure 4 demonstrate, that the mechanical strength is slightly dependent on the building direction and show a tension-compression asymmetry in case of the $90^{\circ}$ specimens from the material conditions $90 \mu \mathrm{m}$ and $30 \mu \mathrm{m}$ (see figures $4 \mathrm{a}$ and $4 \mathrm{~d}$ ). In [11], the tension-compression asymmetry in the $90^{\circ}$ specimens is attributed to the manufacturing process. This is supported by the results of this work, since the asymmetry visible for the $90^{\circ}$ specimens vanishes by introducing a stress relief heat treatment for $2 \mathrm{~h}$ at $300^{\circ} \mathrm{C}$ (compare figures $4 \mathrm{a}$ with $4 \mathrm{~b}$ and $4 \mathrm{~d}$ with $4 \mathrm{e}$ ). If this has anything to do with residual stresses, which usually are known to vanish during LCF loading, remains an open issue. By using finite element crystal plasticity simulations and considering the orientation dependent texture of the same AlSi10Mg alloy, the anisotropy in the elastic stiffness and the yielding behaviour could be explained for tensile tests [51], which show the same tendency as the hysteresis loops in figure 4 .

The pronounced lifetime reduction in the cyclic LCF tests for the as-built specimens is attributed to the rough surface quality and increased defect density close to the surface. Defects with a $\sqrt{\text { area }}$ size of more than $600 \mu \mathrm{m}$ were detected. The proposed fracture mechanics model using $D_{\mathrm{TMF}, \text { brittle }}$ and $\sqrt{\text { area }}$ does not yet account for the detrimental surface effect even if an effective area size is considered (see figures 9 and 10). In [1], the component performance is attributed to surface roughness. The surface roughness is affected by so-called 'staircase effects' [52, 53], whereby the curvature radius of the specimen is approximated stepwise layer by layer. The surface roughness is decreased by a reduced layer thickness [54]. A second effect is addressed to the inhomogeneous melting of individual powder particles or balling networks [1]. As observed in figure 6, the fracture surfaces indicate clearly, that a rough and rugged surface preferably leads to multiple crack initiation and consequently crack coalescence, which can potentially accelerate the damage evolution. According to [8], the lifetime is also decreased in the HCF range for as-built specimens in comparison to machined and heat-treated specimens. An enhanced lifetime behaviour is obtained by a subsequent heat treatment for both layer thicknesses of $30 \mu \mathrm{m}$ and $90 \mu \mathrm{m}$ (see figure 5), which is in accordance with the findings in [8]. A smaller layer thickness also leads to increased LCF lifetimes, which is confirmed in [11] for LCF tests and in [16] for $\mathrm{HCF}$ tests.

The LCF lifetimes of various additively manufactured materials are assessed in [9, 11-13] with the effective cyclic $J$-integral $\Delta J_{\text {eff }}$ expression from Dowling [55]. The approaches with similar formulations for $\Delta J_{\text {eff }}$ have also demonstrated to be successful for lifetime assessment. However, it is not expected that the Dowling formulation with the modifications for internal and surface cracks would automatically lead to a better lifetime prediction for as-built surface specimens, since multiple crack initiation is not taken into account.

The last point, which is discussed, is the final crack length. In the lifetime predictions, the final crack length which determines the end of the fatigue life is chosen to $a_{\mathrm{f}}=a_{0}+1 \mathrm{~mm}$, which is longer than a $1 \mathrm{~mm}$ technical fatigue crack. Since fatigue lives were evaluated according to a load drop criterion, a certain crack growth increment should be required to influence the maximum stress evolution. As in some cases very large initial defect sizes occur, the chosen formulation seems to be appropriate. In [9] the fatigue crack length is set to $3 \mathrm{~mm}$ for the lifetime predictions, as fatigue crack lengths between $2 \mathrm{~mm}$ and $5 \mathrm{~mm}$ were measured on the fracture surfaces. Because the crack growth is quite accelerated at these crack lengths (see e.g. in $[11,37])$ only a negligible number of cycles are accumulated. It is mentioned, that the final crack length could also be further limited by the fracture toughness, which was not considered for reasons of simplicity.

\section{Conclusions}

Low-cycle fatigue tests on additive manufactured AlSi10Mg with different layer thicknesses, heat treatments and surface conditions were performed at room temperature and accompanied by detailed microstructure investigations. The introduction of a solution heat treatment (e.g. within a T6 heat treatment) coarsens the eutectic regions, which form fine, coherent cell structures in the initial state. The mechanical properties and the 
deformation behaviour of the characterized aluminium alloy AlSi10Mg is strongly affected by a subsequent heat treatment. A T5 stress relief heat treatment reduces the mechanical strength significantly. An as-built specimen surface in the initial material condition leads to slightly lower mechanical strength and considerably reduced lifetimes. Subsequent machining and polishing of the specimens is therefore recommended. A reduced powder layer thickness enhances strength and also the lifetime behaviour. The negative surface influence is significantly reduced by a heat treatment. The achieved lifetimes are in good accordance with available SLM and also comparable cast aluminium data from the literature.

High-resolution scanning electron microscopy images were taken from the fracture surface. The defect structure was analysed by means of the $\sqrt{\text { area }}$ parameter. Only the defects, that were responsible for the specimen failure were evaluated. In nearly all cases, manufacturing-related defects such as incompletely melted powder (lack of fusion) were found. Apart from three internal defects, surface or near-surface defects act as crack initiation sites. Especially for the as-built specimens, the fatigue crack is found to initiate at defect clusters. The defect size depends on the building direction, layer thickness and also the surface quality. Compared to the polished specimens, significantly more and larger defects are observed in the as-built specimens, which are prone for multiple crack initiation. For machined and polished specimens, the smallest defects are found for the $45^{\circ}$ orientation. The reduction of the powder layer thickness from $90 \mu \mathrm{m}$ to $30 \mu \mathrm{m}$ also reduces the defect size.

The lifetimes are assessed by means of a mechanismbased lifetime model and the $D_{\mathrm{TMF}, \text { brittle parameter. The }}$ lifetimes show a strong dependence on the initial defect size, since larger defects usually lead to shorter lifetimes. Consequently, the initial defect size is taken into account in a deterministic lifetime model, which allows a significantly improved lifetime prediction. However, the negative surface influence cannot be explained solely by the initial defect size. Therefore, further mechanisms such as multiple crack initiation and the crack coalescence have to be considered in the lifetime model.

\section{Acknowledgements}

The authors greatly acknowledge the financial support of the Fraunhofer-Gesellschaft. Special thanks go to Aron Pfaff, Klaus Hoschke and Martin Jaecklein from the Fraunhofer Ernst-Mach-Institut EMI for manufacturing and preparation of the specimens.

\section{References}

[1] T. DebRoy, H. Wei, J. Zuback, T. Mukherjee, J. Elmer, J. Milewski, A. Beese, A. Wilson-Heid, A. De, W. Zhang, Progress in Materials Science 92, 112 (2018)

[2] E. Olakami, R. Cochrane, K. Dalgarno, Progress in Materials Science 74, 401 (2015)
[3] S. Beretta, S. Romano, International Journal of Fatigue 94, 178 (2017)

[4] D. Buchbinder, Tech. Rep. 01RIO639A-D, Bundesministerium für Bildung und Forschung (2010)

[5] N. Aboulkhair, N. Everitt, I. Ashcroft, C. Tuck, Additive Manufacturing 1-4, 77 (2014)

[6] N. Aboulkhair, M. Simonelli, L. Parry, I. Ashcroft, C. Tuck, R. Hague, Progress in Materials Science 106, 100578 (2019)

[7] E. Brandl, U. Heckenberger, V. Holzinger, D. Buchbinder, Materials and Design 34, 159 (2012)

[8] N. Aboulkhair, I. Maskery, C. Tuck, I. Ashcroft, N. Everitt, Materials and Design 104, 174 (2016)

[9] S. Romano, S. Beretta, S. Foletti, LCF response of AlSilOMg obtained by Additive Manufacturing, in Eighth International Conference on Low Cycle Fatigue (LCF8), Dresden (2017), pp. 53-58

[10] N. Uzan, S. Ramati, R. Shneck, N. Frage, O. Yeheskel, Additive Manufacturing 21, 458 (2018)

[11] S. Romano, L. Patriarca, S. Foletti, S. Beretta, International Journal of Fatigue 117, 47 (2018)

[12] B. Torries, R. Shrestha, A. Imandoust, N. Shamsaei, Fatigue Life Prediction of Additively Manufactured Metallic Materials Using a Fracture Mechanics Approach, in Solid Freeform Fabrication 2018: Proceedings of the 29th Annual International Solid Freeform Fabrication Symposium (2018), pp. 11811190

[13] R. Shrestha, J. Simsiriwong, N. Shamsaei, Additive Manufacturing 28, 23 (2019)

[14] Y. Murakami, Metal fatigue: Effects of Small Defects and Nonmetallic Inclusions, 1 st edn. (Academic Press, 2002)

[15] S. Romano, A. Brandão, J. Gumpinger, M. Gschweitl, S. Beretta, Materials \& Design 131, 32 (2017)

[16] S. Romano, A. Brückner-Foit, A. Brandão, J. Gumpinger, T. Ghidini, S. Beretta, Engineering Fracture Mechanics 187, 165 (2018)

[17] EOS GmbH - Electro Optical Systems, Materialdatenblatt EOS Aluminium AlSi1OMg (2014), downloaded 2017-02-08

[18] B. Flaig, K. Lang, Tech. Rep. 567, Forschungsvereinigung Verbrennungskraftmaschinen e.V. (1994)

[19] I. Maskery, N. Aboulkhair, M. Corfield, C. Tuck, A. Clare, R. Leach, R. Wildman, I. Ashcroft, R. Hague, Materials Characterization 111, 193 (2016)

[20] U. Tradowsky, J. White, R. Ward, N. Read, W. Reimers, M. Attallah, Materials and Design 105, 212 (2016)

[21] N. Takata, H. Kodaira, K. Sekizawa, A. Suzuki, M. Kobashi, Materials Science \& Engineering A 704, 218 (2017)

[22] A. Maamoun, M. Elbestawi, G. Dosbaeva, S.C. Veldhuis, Additive Manufacturing 21, 234 (2018)

[23] X. Cao, J. Campbell, Metallurgical and Materials Transactions A 35, 1425 (2004) 
[24] S. Belmares-Perales, A. Zaldívar-Cadena, Materials Science and Engineering B 174, 191 (2010)

[25] H. Ammar, A. Samuel, F. Samuel, Materials Science and Engineering A 473, 65 (2008)

[26] A. Samuel, F. Samuel, Metallurgical and Materials Transactions A 26, 2359 (1995)

[27] P. Huter, S. Oberfrank, F. Grün, B. Stauder, International Journal of Fatigue 88, 142 (2016)

[28] D. Zhang, Ph.D. thesis, RWTH Aachen (2004)

[29] International Standard ISO 12106, Metallic materials - fatigue testing - Axial-strain-controlled method (2003)

[30] A. Emami, S. Begum, D. Chen, T. Skszek, X. Niu, Y. Zhang, F. Gabbianelli, Materials Science and Engineering A 516, 31 (2009)

[31] S. Michelfeit, Ph.D. thesis, Technische Universität Darmstadt, Fachbereich Maschinenbau (2012)

[32] J. Jordon, M. Horstemeyer, N. Yang, J. Major, K. Gall, J. Fan, D. McDowell, Metallurgical and Materials Transactions A 41, 356 (2010)

[33] D. McDowell, K. Gall, M. Horstemeyer, J. Fan, Engineering Fracture Mechanics 70, 49 (2003)

[34] M. Song, Y. Kong, M. Ran, Y. She, International Journal of Fatigue 33, 1600 (2011)

[35] E. Natesan, S. Eriksson, J. Ahlström, C. Persson, Materials 12, 3033 (2019)

[36] J. Schindelin, I. Arganda-Carreras, E. Frise, V. Kaynig, M. Longair, Nature methods 9, 676 (2012)

[37] C. Fischer, C. Schweizer, S. Mittag, T. Seifert, K. Kuhlen, Tech. Rep. 1188, Forschungsvereinigung Verbrennungskraftmaschinen e.V. (2019)

[38] W. Ramberg, W. Osgood, Tech. Rep. Technical Note No. 902, National Advisory Committee For Aeronautics, Washington DC (1943)

[39] C. Shih, Tech. Rep. MRL E-147, Providence, R.I.: Division of Engineering, Brown University (1983)

[40] C. Schweizer, Ph.D. thesis, Karlsruher Institut für Technologie (KIT), Fakultät für Maschinenbau (2013)

[41] H. Heitmann, H. Vehoff, P. Neumann, in Fracture 84, edited by S. Valluri, D. Taplin, P.R. Rao, J. Knott,
R. Dubey (Pergamon, 1984), pp. 3599 - 3606

[42] M. Metzger, B. Nieweg, C. Schweizer, T. Seifert, International Journal of Fatigue 53, 58 (2012)

[43] W. Schmitt, R. Mohrmann, H. Riedel, A. Dietsche, A. Fischersworring-Bunk, in Fatigue 2002 - Proceedings of the Eighth International Fatigue Congress, edited by A. Blom (Warley, West Midlands: EMAS, 2002), pp. 781-788

[44] J.C. Newman Jr., International Journal of Fracture 24, 131 (1984)

[45] H. Tada, P. Paris, G. Irwin, The Stress Analysis of Cracks Handbook, 3rd edn. (ASME Press, 2000)

[46] M. He, J. Hutchinson, J. Appl. Mech 48, 830 (1981)

[47] H. Riedel, Fracture at high temperatures, 1st edn. (Springer-Verlag Berlin Heidelberg, 1987)

[48] C. Schweizer, T. Seifert, B. Nieweg, P. von Hartrott, H. Riedel, International Journal of Fatigue 33, 194 (2011)

[49] C. Chauvot, M. Sester, Computational Materials Science 19, 87 (2000)

[50] G. Maier, H. Riedel, T. Seifert, J. Klöwer, R. Mohrmann, Time and temperature dependent cyclic plasticity and fatigue crack growth of the nickel-base Alloy617B - experiments and models, in Euro Superalloys 2010 (Trans Tech Publications Ltd, 2011), Vol. 278 of Advanced Materials Research, pp. 369-374

[51] T. Hanemann, Analyse der Mikrostruktur und Simulation der mechanischen Eigenschaften von generativ gefertigtem AlSilOMg (2017), Seminar paper, Karlsruher Institut für Technologie (KIT)

[52] S. Rahmati, E. Vahabli, Int J Adv Manuf Technol 79, 823 (2015)

[53] A. Boschetto, L. Bottini, F. Veniali, Journal of Materials Processing Technology 241, 154 (2017)

[54] C. Qiu, C. Panwisawas, M. Ward, H. Basoalto, J. Brooks, M. Attallah, Acta Materialia 96, 72 (2015)

[55] N. Dowling, in Cyclic stress-strain and plastic deformation aspects of fatigue crack growth (ASTM International, West Conshohocken, 1977), Vol. STP637EB, pp. 97-121 